\title{
COMUNICAÇÃO
}

\section{RESPOSTAS FISIOLÓGICAS DE CAPRINOS DE DIFERENTES GRUPOS GENÉTICOS NO SEMI-ÁRIDO PARAIBANO ${ }^{1}$}

\author{
Fisiological responses of the caprines from different genetic groups in the semi-arid of Paraíba \\ Bonifácio Benicio de Souza ${ }^{2}$, Expedito Danusio de Souza ${ }^{3}$, Rosangela Maria Nunes da Silva ${ }^{4}$, \\ Marcílio Fontes Cezar², José Romulo Soares dos Santos ${ }^{4}$, Gustavo de Assis Silva ${ }^{5}$
}

\begin{abstract}
RESUMO
Realizou-se este experimento na Estação Experimental de Pendência pertencente à EMEPA-PB, localizada no Município de Soledade-PB, com o objetivo de avaliar os parâmetros fisiológicos e hematológicos de caprinos de diferentes grupos genéticos sob as condições do semi-árido. Foram utilizados 30 caprinos machos inteiros, com peso médio de $25,17 \mathrm{~kg}$, sendo seis animais de cada grupo genético: ${ }^{1 / 2}$ Boer + 1/2 SRD (BO), 1/2 Anglo-Nubiana + 1/2 SRD (AN), 1/2 Savana + 1/2 SRD (SA), 1/2 Kalarari + 1/2 SRD (KA) e 1/2 Moxotó + 1/2 SRD (MO). Verificou-se efeito significativo $(\mathrm{P}<0,05)$ de grupos genéticos para a frequiência respiratória e hematócrito. Tendo o BO apresentado frequiência respiratória superior $(\mathrm{P}<0,05)$ ao $\mathrm{AN}$ e $\mathrm{MO}$; e hematócrito inferior $(\mathrm{P}<0,05)$ ao $\mathrm{MO}$. $\mathrm{O}$ turno afetou significativamente $(\mathrm{P}<0,05)$ a temperatura retal e a freqüência respiratória, com médias superiores à tarde. Para as demais variáveis não houve diferença significativa entre os grupos genéticos estudados. Permitindo concluir que os animais estudados estão bem adaptados ao clima semi-árido.
\end{abstract}

Termos para indexação: Cabras, raça, estresse, clima, adaptabilidade.

\begin{abstract}
This experiment was realized in Pendencia's experimental station of EMEPA-PB in Soledade- PB city. It aimed to avaliate the fisiological and hematological parameters of caprines from diferent genetic groups in the semi-arid conditions. Thirty males weithing about $25,17 \mathrm{~kg}$ were used, being six animals from each genetic group: $1 / 2$ Boer $+1 / 2$ SRD (BO), $1 / 2$ Anglo-Nubiana $+1 / 2$ SRD (AN), $1 / 2$ Savana $+1 / 2$ SRD (SA), $1 / 2$ Kalarari $+1 / 2$ SRD (KA) e 1/2 Moxotó $+1 / 2$ SRD (MO). It was verified significative effect $(\mathrm{P}<0,05)$ among the genetic groups for breathing frequency and hematocrit. The BO presented breathing frequency more elevated $(\mathrm{P}<0,05)$ than the AN and MO; and its hematocrit was less elevated $(\mathrm{P}<0,05)$ than the MO. The period affects sifgnificatively $(\mathrm{P}<0,05)$ the rectal temperature and breathing frequency, being the superior averages in the afternoon period. For the other parameters there were not significative differences among the genetic groups studied. Besides this, it can be concluded that the studied animals are adaptaded to the semi-arid climatic conditions.
\end{abstract}

Index terms: Goats, breed, stress, climatic, adaptability.

\section{(Recebido em 18 de maio de 2006 e aprovado em 21 de março de 2007)}

O conhecimento das variáveis climáticas, sua interação com os animais e as respostas comportamentais, fisiológicas e produtivas são preponderadas na adequação do sistema de produção aos objetivos da atividade. Dessa forma a interação animal-ambiente deve ser considerada, quando se busca maior eficiência na exploração pecuária. As diferentes respostas do animal às peculiaridades de cada região, são determinantes para o sucesso da atividade através da adequação do sistema produtivo às características do ambiente e ao potencial produtivo dos ruminantes (TEIXEIRA, 2000).

A temperatura ambiente representa a principal influência climatológica sobre as variáveis fisiológicas, frequiência respiratória e temperatura retal, seguida em ordem de importância pela radiação solar, umidade relativa do ar e o movimento do ar. Também tem sido objeto de estudo a freqüência cardíaca e os constituintes sangüíneos (LEE et al., 1974).

\footnotetext{
${ }^{1}$ Parte da dissertação do segundo autor - financiada pelo CNPq/Capes

${ }^{2}$ Doutores, Professores - Unidade Acadêmica de Medicina Veterinária/UAMV - Universidade Federal de Campina Grande/UFCG - Centro de Saúde e Tecnologia Rural/CSTR - Campus de Patos - Cx. P. 64 -58700-000 - bonif@cstr.ufcg.edu.br; mfcezar@gmail.com

${ }^{3}$ Mestre - Escola Agrotécnica Federal de Iguatu - Cx. P. 38 -63500-00 - Iguatu, CE - danusiosouza@yahoo.com.br

${ }^{4}$ Mestres - Unidade Acadêmica de Medicina Veterinária/UAMV - Universidade Federal de Campina Grande/UFCG - Centro de Saúde e Tecnologia Rural/ CSTR - Campus de Patos - Cx. P. 64 -58700-000 - mnsilva@bol.com.br; jromulosmedicoveterinario@bol.com.br

${ }^{5}$ Mestre - Instituto de Pesquisa Agropecuária/IPA - Avenida Conselheiro João Alfredo, 56, sala 1 - 55330-000 - Bom Conselho, PE gugavet2000@yahoo.com.br
} 
O impacto do calor sobre as variáveis fisiológicas resulta em um aumento percentual de 3,3\% na temperatura retal e $194 \%$ na requiência respiratória, com alterações, respectivamente, de $38,6^{\circ} \mathrm{C}$ para $39,9^{\circ} \mathrm{C}$ e de 32 para 94 mov/min (MCDOWELL, 1972). A freqüência respiratória alta pode ser uma maneira eficiente de perder calor por curtos períodos, mas quando mantida por várias horas, poderá resultar em sérios problemas para os animais. A respiração acelerada e contínua pode interferir na ingestão de alimentos e ruminação, adicionar calor endógeno a partir da atividade muscular e desviar a energia que poderia ser utilizada em outros processos metabólicos e produtivos. A temperatura corporal é o resultado do equilíbrio entre energia térmica produzida e energia térmica dissipada. Um aumento na TR significa que o animal está estocando calor, e se este não é dissipado, o estresse calórico manifesta-se.

De acordo com Paes et al. (2000) os parâmetros sangüíneos têm sido utilizados mundialmente para avaliar o estado de saúde dos animais e também como indicadores de estresse calórico. Entretanto, outros fatores podem influenciar os valores de referência para a interpretação dos referidos parâmetros, tais como: espécie, sexo, raça, idade, estado fisiológico e hora do dia (JAIN, 1993).

Archer (1967), cita como valores normais para caprinos: hemoglobina $11(8-14) \mathrm{g} / 100 \mathrm{ml}$; volume globular $34(29-38) \%$ e leucócitos $9000(5-13000) \mathrm{mm}$.

Bhargava (1980), trabalhando com 50 caprinos clinicamente sadios obteve os seguintes resultados: hemoglobina 10,09 (7,1-11,2 G/100ml; hematócrito 31, 96

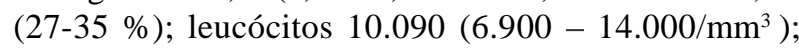
segmentados 39 (28-66\%); linfócitos 57,7 (29-69\%) monócitos 2,12(1-4\%).

Holman \& Dew (1963), estudando o hemograma de caprinos com 2 a 3 anos de idade, mantidos em confinamento, encontraram os seguintes resultados: hemácias $\left(10^{6} / \mathrm{mm}^{3}\right)$ 12,73 $\pm 2,63$, volume globular (\%) 28,66 $\pm 4,57$, hemoglobina (g\%) 11,09 $\pm 1,78$, CHGM, VGM $\left(^{3}\right)$ $22,69 \pm 3,74$. Em outro experimento com animais jovens (ao nascimento) e adultos (30 meses) encontraram o seguinte: hemácia $\left(10^{6} / \mathrm{mm}^{3}\right) 9,3$ e 11,3 , volume globular (\%) 42 e 28 e VGM $\left(\mathrm{m}^{3}\right) 45$ e 25 (HOLMAN \& DEW, 1964).

Oduye (1976), na Nigéria, trabalhando com caprinos adultos e jovens, suplementados com concentrado durante todo o ano, registrou os seguintes valores: Leucócitos $\left(10^{3} / \mathrm{mm}^{3}\right) 7,55 \pm 4,10$, Neutrófilos 44,9\% \pm 7,60, Bastonetes $0,2 \% \pm 0,40$, Eosinófilos $4,3 \% \pm 4,60$, Linfócitos $50,2 \% \pm$ 7,4 e Monócitos 1,0\% — 0,9.

Segundo Jain (1986) os valores eritrocitários normais determinados para caprinos são hemácias 8 a 18 x 106/ml; Hb 8 a 12g/dl; VG 22 a 38\%; VCM 16 a 25 fl; HCM 5,2 a 8,0pg e CHCM a 36\%. Para Lee et al. (1974), a variação do volume globular depende da severidade da carga calórica imposta sobre o animal. Um estresse por calor de longa duração em bovinos pode reduzir o número de eritrócitos e o volume globular, tendo sido atribuído a uma hemoconcentração, em função da diminuição da ingestão de alimentos.

Herz \& Steinhaut (1978) relatam que um estresse térmico de longa duração em bovinos e ovinos provoca uma diminuição no percentual do hematócrito. Segundo Somvanshi et al. (1987), em caprinos neonatos (até 30 dias de idade) os valores de hemoglobina e volume globular são ligeiro a moderadamente mais elevados do que nas idades de 6 a 9 meses, 3 a 5 anos e 6 a 10 anos. A contagem total de leucócitos eleva-se a partir do nascimento em razão doaumento do número absoluto de linfócitos e atinge o pico máximo por volta dos 3 a 4 meses. Nesse momento, inicia-se o declíneo até atingir o valor de adulto que está próximo ao do nascimento. A média da contagem leucocitária em caprinos foi de $16,5 \times 10^{3} / \mathrm{ml}$ no quarto mês, e depois variou entre 12 e $15 \times 10^{9} / \mathrm{L}$ (GREENWOOD, 1977).

Os valores de leucócitos totais relatados por Jain (1986) para caprinos normais foram de $4-13 \times 10^{3} / \mathrm{ml}$, neutrófilos de 1,3-7,2 × $10^{3} / \mathrm{ml}$, linfócitos de 2,0-9,0 × $10^{3}$ $/ \mathrm{ml}$; monócitos de $0-550 / \mathrm{ml}$ eosófilos de $50-650 / \mathrm{ml}$ e basófilos de $0-12 / \mathrm{ml}$.

Os animais criados sob diferentes condições climáticas podem apresentar evidentes variações dos elementos constituintes do hemograma. Assim, os valores obtidos para os animais criados em uma região não podem ser considerados, sem uma adequada avaliação, como padrão de referência fora dessa região (BIRGEL JÚNIOR et al., 2001). Portanto, torna-se necessária a determinação do eritrograma das raças exploradas na região semi-árida, tendo em vista que a temperatura ambiente nessa região, durante a maior parte do ano, é bastante elevada.

Objetivou-se neste trabalho avaliar as respostas fisiológicas e os parâmetros hematológicos de caprinos de diferentes grupos genéticos no semi-árido paraibano.

O experimento foi realizado na Estação Experimental de Pendência pertencente à Empresa Estadual de Pesquisa Agropecuária da Paraíba S/A (EMEPA), localizada no Município de Soledade-PB, na microrregião do Curimatau Ocidental, no Agreste Paraibano. O clima da região é quente e seco do tipo semi-árido subtipo BSW'h, caracterizandose por apresentar um período de estiagem de 5 a 6 meses (SILVA et al., 1987), com temperatura máxima anual de $35^{\circ} \mathrm{C}$ e a mínima é de $22^{\circ} \mathrm{C}$. A umidade relativa do ar situa-se em torno de $50 \%$ e a precipitação pluviométrica apresenta uma amplitude com variação de 104 à 705 mm/ano, e média geral em torno de 390 mm/ano (MEDEIROS, 1996). 
Os animais do experimento foram obtidos pelo programa de melhoramento para caprinos de corte, desenvolvido pela EMEPA, com o apoio do Banco do Nordeste e do CNPq, utilizando os recursos genéticos disponíveis na região, mediante estratégias de cruzamento. Foram utilizados 30 caprinos machos inteiros, com peso médio de $25,17 \mathrm{~kg}$, sendo seis animais de cada grupo genético $1 / 2$ Boer + 1/2 SRD (BO), $1 / 2$ Anglo-Nubiana + 1/2 SRD (AN), $1 / 2$ Savana + 1/2 SRD (SA), $1 / 2$ Kalarari + 1/2 SRD (KA) e $1 / 2$ Moxotó + 1/2 SRD (MO). A dieta foi composta por feno de maniçoba (Manihot glaziowii), forrageira nativa da caatinga, com uma mistura concentrada (milho, soja, óleo vegetal e sal mineral), formulada visando obter-se uma dieta com alto valor nutritivo $73,1 \%$ de NDT e $18,6 \%$ de PB. Os dados da dieta concentrada e de volumoso encontram-se na Tabela 1.

Tabela 1 - Composição da ração concentrada e do feno em termos de participação percentual dos ingredientes.

\begin{tabular}{lc}
\hline \multicolumn{1}{c}{ Ingrediente } & Percentual \\
\hline Concentrado & \\
Farelo de milho & $50 \%$ \\
Farelo de soja & $23 \%$ \\
Farelo de trigo & $5 \%$ \\
Óleo vegetal & $1 \%$ \\
Sal mineral & $1 \%$ \\
Volumoso & \\
Feno de maniçoba & $20 \%$ \\
\hline Total & $100 \%$ \\
\hline
\end{tabular}

Durante a fase experimental foram registradas as seguintes variáveis ambientais: temperatura máxima (Tmáx) e temperatura mínima (Tmín); temperatura do bulbo seco (Tbs) e temperatura do bulbo úmido (Tbu); umidade relativa (UR); temperatura do globo negro (Tgn) e calculado o índice de temperatura e umidade (ITGU) de acordo com a fórmula: ITGU $=$ Tgn $+0,36$ (Tpo) + 41,5 (BUFFINGTON et al., 1981). As leituras foram realizadas em três dias alternados por semana, durante as quatro semanas do mês de setembro de 2002.

Os parâmetros avaliados foram: temperatura retal (TR), frequiência respiratória (FR) e freqüência cardíaca (FC) hematócrito (Ht), volume globular médio (VGM), hemoglobina globular média (HGM) e da concentração de hemoglobina globular média (CHGM), concentração de leucócitos (LEUC), percentagem de segmentados (SEGM), linfócitos (LINF) e monócitos (MON).
Para os parâmetros fisiológicos utilizou-se o delineamento inteiramente casualizado, com seis repetições, em parcelas subdivididas no tempo (STELL \& TORRIE, 1980). As parcelas foram constituídas dos cinco grupos genéticos e as subparcelas pelos turnos (manhã e tarde). As médias foram comparadas pelo teste de Tukey, a $1 \%$ e a $5 \%$ de probabilidade. E para os parâmetros hematológicos foi utilizado o delineamento inteiramente casualizado, com cinco tratamentos (grupos genéticos) e seis repetições.

A metodologia aplicada para obtenção da temperatura retal (TR) consistiu na introdução de um termômetro clínico veterinário, com escala até $44^{\circ} \mathrm{C}$, diretamente no reto do animal, a uma profundidade de 5 $\mathrm{cm}$, de forma que o bulbo ficasse em contato com a mucosa do animal, permanecendo por um período de dois minutos e o resultado da leitura expresso em graus centígrados $\left({ }^{\circ} \mathrm{C}\right)$ (BACCARI JÚNIOR, 1990).

Para análise hematológica foi coletada uma amostra de $7 \mathrm{ml}$ de sangue de cada animal experimental em vacutainer contendo anticoagulante EDTA (etilenodiaminoacetato), onde foram imediatamente centrifugados em tubos de ensaios a 3000 rpm para completa retração do coágulo. A fase líquida obtida, o soro sanguíneo, foi acondicionada em freezer para posterior análise. O estudo do perfil hematológico foi realizado no Laboratório de Patologia Clínica Veterinária da Universidade Federal de Campina Grande - Campus de Patos, PB, através da hematimetria e leucometria. Na primeira avaliação, foram determinadas a taxa de hemoglobina ( $\mathrm{gr} / \mathrm{dl})$ pelo método da cianometahemoglobina, o volume globular (\%) pela técnica do microhematócrito e os índices hematimétricos absolutos (VGM, HGM e CHGM). Na segunda avaliação, foram medidas, através de esfregaço sanguíneo e diferenciação, a concentração de leucócitos/mm3 e a porcentagem de segmentados, bastonetes, mielócitos, metamielócitos, eosinófilos, basófilos, linfócitos e monócitos.

A análise de variância foi realizada utilizando-se o programa Sistema de Análises Estatísticas e Genéticas (UFV, 1993), e as médias comparadas pelo teste de Tukey, a $5 \%$ de probabilidade.

Os dados climatológicos registrados durante o experimento encontram-se na Tabela 2. Onde observa-se que a Tbs nos turnos manhã e tarde encontra-se dentro da Zona de Conforto Térmico, que, para caprinos, deve ficar entre 20 e $30^{\circ} \mathrm{C}$ (BAETA \& SOUZA, 1997), no turno da manhã a Tbs de $21,28^{\circ} \mathrm{C}$ e UR de $61 \%$ estão dentro da situação de conforto, já no turno da tarde a Tbs de $28,85^{\circ} \mathrm{C}$ e UR de $41 \%$ caracteriza uma situação de perigo, segundo o U. S. Weather Bureau (MOTA, 2001). 
Tabela 2 - Média das variáveis ambientais e o cálculo do índice de temperatura do globo negro e umidade (ITGU).

\begin{tabular}{lccc}
\hline \multicolumn{1}{c}{ Variáveis ambientais } & \multicolumn{2}{c}{ Turnos } & Media diária \\
\cline { 2 - 4 } & Manhã & Tarde & 31,86 \\
\hline Temperatura máxima, Tmáx $\left({ }^{\circ} \mathrm{C}\right)$ & - & - & 20,00 \\
Temperatura mínima, Tmín $\left({ }^{\circ} \mathrm{C}\right)$ & - & - & 25,06 \\
Temperatura do bulbo seco, Tbs $\left({ }^{\circ} \mathrm{C}\right)$ & 21,28 & 28,85 & 17,89 \\
Temperatura do bulbo úmido, Tbu $\left({ }^{\circ} \mathrm{C}\right)$ & 16,28 & 19,50 & 51,00 \\
Umidade relativa, UR $(\%)$ & 61,00 & 41,00 & 28,57 \\
Temperatura do globo negro, Tgn $\left({ }^{\circ} \mathrm{C}\right)$ & 24,71 & 32,43 & 75,20 \\
Índice de temperatura do globo negro e umidade, ITGU & 71,25 & 79,15 & \\
\hline
\end{tabular}

A temperatura do globo negro, determinada pela equipe do Conforto Ambiental da UNICAMP, é considerada regular de $27-34{ }^{\circ} \mathrm{C}$ e acima de $35{ }^{\circ} \mathrm{C}$ é considera crítica (MOTA, 2001).

Nesse, caso observa-se que os valores da temperatura do globo negro tanto no turno da manhã quanto no turno da tarde estão dentro do intervalo considerado regular para o conforto ambiental.

O ITGU, pela manhã, foi de 71,25 e a tarde de 79,15. De acordo com o National Weather Service U.S., citado por Baêta (1985), os valores de ITGU até 74 definem situação de conforto; de 74 a 78 situação de alerta; de 79 a 84 situação perigosa, e acima de 84 emergência para vaca leiteiras. Considerando que o ITGU, na parte da tarde, de 79,15 representa para vacas leiteiras um estresse calórico elevado, para os caprinos estudados não teve o mesmo efeito, provavelmente em função alta capacidade de dissipação de calor dos mesmos, pois a TR dos diferentes grupos genéticos se mostraram dentro da normalidade, de acordo com Brion (1964) que considera normal uma variação de 39 a $40{ }^{\circ} \mathrm{C}$.

A análise de variância não demonstrou efeito significativo de grupo genético para os parâmetros TR e FC. Contudo, para FR foi verificado efeito significativo $(\mathrm{P}<0,05)$ tendo o BO apresentado a FR superior $(\mathrm{P}<0,05)$ ao AN e MO (Tabela 3). Com relação ao turno, verificou-se efeito significativo $(\mathrm{P}<0,05)$ para a TR e FR, com médias superiores no período da tarde. Resultados que corroboram com Silveira (1999) que, trabalhando com caprinos das raças Boer e Anglo-Nubiana no semi-árido paraibano, verificou que os animais da raça Boer demonstraram freqüência respiratória significativamente $(\mathrm{P}<0,05)$ mais elevada do que os da raça Anglo-Nubiana.

A análise de variância revelou efeito significativo $(\mathrm{P}<0,05)$ para a variável hematócrito $(\mathrm{Ht})$, mas não houve efeito $(\mathrm{P}>0,05)$ para as demais variáveis hematimétricas, conforme notam-se pela Tabela 4 .

Não houve diferença significativa $(\mathrm{P}>0,05)$ para os resultados de hemoglobina entre os diferentes grupos genéticos (Tabela 4). Esses resultados são inferiores aos relatados por Paes et al. (2000), que realizaram trabalho com caprinos fêmea da raça Parda Alpina de diferentes idades, porem, assemelha-se à média de $8,29 \mathrm{~g} / 100 \mathrm{ml}$, que foi encontrada por Matos et al. (1982) em experimento com caprinos.

Não houve diferença significativa $(\mathrm{P}>0,05)$ para a variável hematócrito entre os grupos genéticos $1 / 2$ Savana $+1 / 2$ SRD, $1 / 2$ Kalahari + $1 / 2$ SRD e $1 / 2$ Anglo Nubiana + 1/2 $\mathrm{SRD}$, mas houve diferença significativa $(\mathrm{P}<0,05)$ para os grupos $1 / 2$ Boer $+1 / 2$ SRD e $1 / 2$ Moxotó $+1 / 2$ SRD. A variação do hematócrito depende da severidade da carga calórica imposta sobre o animal (BIANCA, 1965). Segundo Lee et al. (1974) o hematócrito pode aumentar em função de uma desidratação, por causa da perda de líquidos através dos mecanismos evaporativos de dissipação de calor, como respiração e sudorese.

Não foi encontrada diferença significativa entre os grupos genéticos para as variáveis: volume globular médio (VGM), hemoglobina globular média (HGM) e para concentração de hemoglobina globular média (CHGM). Trabalhando com fêmeas jovens de 4 meses, Paes et al. (2000), encontraram valor máximo para volume globular médio de 20,83 fl, hemoglobina globular média 7,67 pg e para concentração de hemoglobina globular média $39,63 \%$.

Com os resultados encontrados, conclui-se que os grupos genéticos estudados respondem fisiologicamente bem ao clima semi-árido. 
Tabela 3 - Médias e desvios-padrão das variáveis fisiológicas: temperatura retal (TR), frequiência respiratória (FR) e freqüência cardíaca (FC), dos diferentes grupos genéticos estudados.

\begin{tabular}{cccc}
\hline Grupo genético & TR $\left({ }^{\circ} \mathrm{C}\right)$ & FR $(\mathrm{mov} / \mathrm{min})$ & FC $(\mathrm{bat} / \mathrm{min})$ \\
\hline BO & $39,58 \pm 0,19 \mathrm{a}$ & $58,67 \pm 14,22 \mathrm{a}$ & $126,32 \pm 10,04 \mathrm{a}$ \\
SA & $39,61 \pm 0,23 \mathrm{a}$ & $52,61 \pm 10,86 \mathrm{ab}$ & $133,06 \pm 9,60 \mathrm{a}$ \\
KA & $39,60 \pm 0,18 \mathrm{a}$ & $54,47 \pm 11,83 \mathrm{ab}$ & $123,36 \pm 10,90 \mathrm{a}$ \\
AN & $39,43 \pm 0,30 \mathrm{a}$ & $45,47 \pm 16,02 \mathrm{~b}$ & $124,75 \pm 11,39 \mathrm{a}$ \\
MO & $39,63 \pm 0,28 \mathrm{a}$ & $45,95 \pm 11,77 \mathrm{~b}$ & $121,36 \pm 10,80 \mathrm{a}$ \\
\hline Turnos & $39,46 \pm 0,20 \mathrm{~b}$ & $42,30 \pm 8,56 \mathrm{~b}$ & $127,96 \pm 10,34 \mathrm{a}$ \\
Manhã & $39,68 \pm 0,23 \mathrm{a}$ & $60,57 \pm 11,48 \mathrm{a}$ & $125,18 \pm 11,36 \mathrm{a}$ \\
\hline Tarde & 0,47 & 15,43 & 4,31
\end{tabular}

Médias seguidas de mesma letra não diferem significamente pelo teste de Tukey a $5 \%$ de probabilidade.

Tabela 4 - Valores médios do número hematócrito (Ht), volume globular médio (VGM), hemoglobina globular média (HGM) e da concentração de hemoglobina globular média (CHGM), de caprinos de diferentes grupos genéticos no semi-árido.

\begin{tabular}{cccccc}
\hline \multirow{2}{*}{$\begin{array}{c}\text { Grupo } \\
\text { genético }\end{array}$} & $\mathrm{Hb}(\mathrm{g} / \mathrm{dl})$ & $\mathrm{HT}(\%)$ & $\mathrm{VGM}\left(\mu^{3}\right)$ & $\mathrm{HGM}(\mu \mu \mathrm{g})$ & $\mathrm{CHGM}(\%)$ \\
\cline { 2 - 6 } & $8,22 \pm 0,99 \mathrm{a}$ & $21,17 \pm 4,71 \mathrm{~b}$ & $18,05 \pm 2,3 \mathrm{a}$ & $7,18 \pm 3,13 \mathrm{a}$ & $40,04 \pm 6,85 \mathrm{a}$ \\
$\mathrm{BO}$ & $8,25 \pm 0,56 \mathrm{a}$ & $23,17 \pm 1,72 \mathrm{ab}$ & $18,36 \pm 2,79 \mathrm{a}$ & $6,54 \pm 0,97 \mathrm{a}$ & $35,69 \pm 2,38 \mathrm{a}$ \\
$\mathrm{SA}$ & $8,25 \pm 1,34 \mathrm{a}$ & $23,17 \pm 3,66 \mathrm{ab}$ & $20,91 \pm 2,96 \mathrm{a}$ & $7,44 \pm 0,95 \mathrm{a}$ & $35,63 \pm 0,92 \mathrm{a}$ \\
KA & $8,16 \pm 1,11 \mathrm{a}$ & $22,67 \pm 2,25 \mathrm{ab}$ & $20,84 \pm 2,91 \mathrm{a}$ & $7,46 \pm 0,89 \mathrm{a}$ & $36,16 \pm 4,92 \mathrm{a}$ \\
NA & $9,88 \pm 1,59 \mathrm{a}$ & $28,17 \pm 3,71 \mathrm{a}$ & $21,99 \pm 3,17 \mathrm{a}$ & $7,72 \pm 1,36 \mathrm{a}$ & $35,11 \pm 3,85 \mathrm{a}$ \\
\hline
\end{tabular}

Médias seguidas de mesma letra não diferem significativamente pelo teste Tukey a $5 \%$ de probabilidade.

Tabela 5 - Valores médios do número da concentração de leucócitos (LEUC), percentagem de segmentados (SEGM), linfócitos (LINF) e monócitos (MON) de caprinos de diferentes grupos genéticos no semi-árido.

\begin{tabular}{ccccc}
\hline \multirow{2}{*}{$\begin{array}{c}\text { Grupo } \\
\text { genético }\end{array}$} & LEUC $\left(10^{3} / \mathrm{mm}^{3}\right)$ & SEGM $(\%)$ & LINF $(\%)$ & MON $(\%)$ \\
\cline { 2 - 5 } & $12108,33 \pm 4,46 \mathrm{a}$ & $24,83 \pm 13,30 \mathrm{a}$ & $74,33 \pm 13,03 \mathrm{a}$ & $0,67 \pm 0,82 \mathrm{a}$ \\
BO & $12375,00 \pm 1,73 \mathrm{a}$ & $33,00 \pm 10,58 \mathrm{a}$ & $66,83 \pm 11,13 \mathrm{a}$ & $0,83 \pm 1,60 \mathrm{a}$ \\
SA & $14525,00 \pm 3,44 \mathrm{a}$ & $27,50 \pm 10,69 \mathrm{a}$ & $70,67 \pm 11,25 \mathrm{a}$ & $1,50 \pm 1,05 \mathrm{a}$ \\
KA & $15525,00 \pm 3,17 \mathrm{a}$ & $18,00 \pm 3,22 \mathrm{a}$ & $79,83 \pm 3,31 \mathrm{a}$ & $2,00 \pm 1,67 \mathrm{a}$ \\
NA & $17425,00 \pm 2,53 \mathrm{a}$ & $31,00 \pm 6,54 \mathrm{a}$ & $68,00 \pm 6,54 \mathrm{a}$ & $0,50 \pm 0,84 \mathrm{a}$ \\
\hline
\end{tabular}

Médias seguidas de mesma letra não diferem significativamente pelo teste Tukey a $5 \%$ de probabilidade.

\section{REFERÊNCIAS BIBLIOGRÁFICAS}

ARCHER, R. K. Técnicas de hematologia animal. Zaragoza: Acríbia, 1967. 164 p.
BAÊTA, F. C. Responses of lacting dairy cows to the combined effects of temperature, humidity and wind velocity in the warm season. 1985. $281 \mathrm{f}$. Thesis (Ph.D.) University Missouri, Missouri, 1985. 
BACCARI JÚNIOR, F. Métodos e técnicas de avaliação da adaptabilidade dos animais às condições tropicais. In: SIMPÓSIO INTERNACIONAL DE BIOCLIMATOLOGIA ANIMAL NOS TRÓPICOS: PEQUENOS E GRANDES RUMINANTES, 1., 1990, Sobral, CE. Anais... Sobral: Embrapa-CNPC, 1990. p. 9-17.

BAÊTA, F. C.; SOUZA, C. de F. Ambiência em edificações rurais: conforto animal. Viçosa: UFV, 1997. 246 p.

BHARGAVA, S. C. Hematological studies in goat. Indian Veterinary Journal, New Delhi, v. 57, n. 6, p. 485-486, 1980.

BIANCA, W. Reviews of the progress of dairy science. Journal Dairy Research, Cambridge, n. 32, p. 291-345, 1965.

BIRGEL JUNIOR, E. H.; D’ANGELO, J. L.; BENESI, F. J. Valores de referência do eritrograma de bovinos da raça Jersey criados no estado de São Paulo. Arquivo Brasileiro de Medicina Veterinária e Zootecnia, Belo Horizonte, v. 53, n. 2, p. 164-171, 2001.

BRION, A. Vademecum del veterinário. 2. ed. Barcelona: Gea, 1964. 732 p.

BUFFINGTON, D. E.; COLLAZO-AROCHO, A.; CANTON, G. H.; PITT, D.; THATCHER, W. W.; COLLIER, R. J. Black globe-humidity index (BGHI) as comfort equation for dairy cows. Transactions of the ASAE, Michigan, v. 24, n. 3, p. 711-714, May/June 1981.

GREENWOOD, B. Hematology of the sheep and goat. In: ARCHER, L. K.; JEFFCOTT, L. B. Comparative clinical haematology. Oxford: Blackwell Scientific, 1977. p. 305.

HERZ, A.; STEINHAUT, D. The reaction of domestic animal to heat stress. Animal Research Development, [S.1.], n. 7, p. 7-38, 1978.

HOLMAN, H. H.; DEW, S. M. The blood picture of the goat: I. the two year old female goat. Research Veterinary Science, Oxford, v. 4, n. 1, p. 121-130, 1963.

HOLMAN, H. H.; DEW, S. M. The blood picture of the goat: II. changes in erythocytic shape, size and number associated with age. Research Veterinary Science, Oxford, v. 5, n. 3, p. 274-285, 1964.

JAIN, N. C. Essentials of veterinary hematology. Philadelphia: Lea \& Febinger, 1993. 417 p.
JAIN, N. C. Schalm's veterinary hematology. 4. ed. Philadelphia: Lea \& Febinger, 1986. 1221 p.

LEE, J. A.; ROUSSEL, J. D.; BEATTY, J. F. Effect of temperature season on bovine adrenal cortical function, blood cell profile, and milk production. Journal of Dairy Science, Cambridge, v. 59, n. 1, p. 104-108, 1974.

MATOS, M. S.; SOUZA, R. M. da; SANTOS, L. M. M. dos; RIBEIRO, O. C.; SANTOS, J. A. C.; BORGES, W. Hemoglobina, volume globular e leucócitos em caprinos. Arquivo da Escola de Medicina Veterinária da Universidade Federal da Bahia, Salvador, v. 7, n. 1, p. 82-90, 1982.

MCDOWELL, R. G. Improvement of livestock production in warm cllimates. San Francisco: Freeman, 1972. 711 p.

MEDEIROS, G. R. Peso a cobrição, ganho de peso durante a gestação e prolificidade de cabras nativas, exóticas e mestiças no semi-árido. 1996. 50 f. Dissertação (Mestrado) - Universidade Federal da Paraíba, Areia, 1996.

MOTA, F. S. Climatologia zootecnica. Pelotas: UFPel, 2001. $104 \mathrm{p}$.

ODUYE, O. O. Hematological values of Nigerian goats and sheep. Tropical Animal Heaith Production, Edimburgh, v. 8, n. 3, p. 131-136, 1976.

PAES, P. R.; BAIRONI, G.; FONTEQUE, J. R. Comparação dos valores hematológicos entre caprinos fêmeas da raça Parda Alpina de diferentes faixas etárias. Veterinária Notícias, [S.1.], v. 6, n. 1, p. 43-49, 2000.

SILVA, M. A. V.; BRAGA, C. C.; NIETZSCHE, M. H. Atlas climatológico do Estado da Paraíba. 2. ed. Campina Grande: UFPB, 1987.

SILVEIRA, J. O. de A. Respostas adaptativas de caprinos das raças Boer e Anglo-Nubiana às condições do semiárido brasileiro. 1999. 37 f. Dissertação (Mestrado) Universidade Federal da Paraíba, Areia, 1999.

SOMVANSHI, R.; BISWAS, J. C.; SHARMA, B.; KOUL, G. L. Haematological studies on Indian pashmina goats. Research Veterinary Science, Oxford, v. 42, p. 124-126, 1987.

SOUSA, W. H. de. Programa de melhoramento dos caprinos de corte no nordeste do Brasil e suas perspectivas. In: SIMPÓSIO NACIONAL DE MELHORAMENTO ANIMAL, 4., 2002, Campo Grande, MS. Anais... Campo Grande: Embrapa-CNPGC, 2002. 
STEEL, R. D. G.; TORRIE, J. H. Principles and procedures of statistics: biometria approach. 2. ed. New York: McGrawHill Book, 1980. 633 p.

TEIXEIRA, M. Efeito do estresse climático sobre parâmetros fisiológicos e produtivos em ovinos. 2000. 62 f.
Dissertação (Mestrado) - Universidade Federal do Ceará, Fortaleza, 2000.

UNIVERSIDADE FEDERAL DE VIÇOSA. SAEG Sistema de Análises Estatísticas e Genéticas. Viçosa, 1993. 